\title{
ANAESTHETIC MANAGEMENT OF A GERIATRIC PATIENT WITH PARKINSON'S DISEASE AND DIABETES MELLITUS POSTED FOR EMERGENCY LAPAROTOMY
}

Prajwal Patel H. S' ${ }^{1}$, Shivaramu B. T², Vidyadhar Metri³, Amith S4, Kislaya Kumar ${ }^{5}$

\section{HOW TO CITE THIS ARTICLE:}

Prajwal Patel H. S, Shivaramu B. T, Vidyadhar Metri, Amith S, Kislaya Kumar. "Anaesthetic Management of a Geriatric Patient with Parkinson`S Disease and Diabetes Mellitus Posted for Emergency Laparotomy". Journal of Evolution of Medical and Dental Sciences 2014; Vol. 3, Issue 51, October 09; Page: 12048-12052,

DOI: $10.14260 /$ jemds/2014/3591

ABSTRACT: Parkinson's disease (PD), one of the most common disabling neurological diseases, affects about $1 \%$ of the population over 60 years of age. It is a degenerative disease of the central nervous system caused by the loss of dopaminergic fibers in basal ganglia of the brain. PD is an important cause of perioperative morbidity and with an increasingly elderly population; it is being encountered with greater frequency in surgical patients. Here we report a case of 79year old male with Parkinsonism and diabetes mellitus posted for emergency laparotomy, which we managed successfully with general anesthesia.

KEYWORDS: Parkinson disease, Diabetes mellitus, Laparotomy, Geriatric.

INTRODUCTION: PD occurs worldwide affecting all ethnic age groups. Parkinson's disease affects $>1$ million individuals in the United States. Its peak age of onset is in sixties (35-65 years) and the course of illness ranges from 10 to 25 years. Approximately $0.3 \%$ of the general population and $3 \%$ of the population above 65 years have PD. ${ }^{1}$ Parkinsonism is the name given to a clinical syndrome comprising impairment of voluntary movement (hypokinesia), rigidity, and tremor.

Because of the characteristic stooped posture and chaplinesque shuffling, Parkinson's disease (PD) has been called "the happy disease". The characteristic pathological feature is the destruction of dopamine-containing nerve cells in sub stantia nigra of basal ganglia. ${ }^{1}$ Particular an aesthetic problems in PD include old age, anti-parkinsonism a drug interaction with anaesthetic drugs and various alterations in the respiratory, cardiovascular, autonomic, and neurological systems. ${ }^{2}$

CASE REPORT: A 79 year old male, who weighed $45 \mathrm{~kg}$ and had Parkinson`s disease was scheduled for emergency explorative laparotomy for a suspected acute intestinal perforation .He had been diagnosed as PD 10 years ago and was on irregular treatment with oral tablets, tab. Levodopa 200mg daily. Patient is also known diabetic since 30 years on irregular treatment with oral hypoglycemic tab metformin 500mg daily and has not consumed them since 36 hours.

There was history of bladder dysfunction in the form of increased urinary frequency and urgency there was no history of any other long term medication or drug abuse or any psychiatric illness. There was no history of any previous anaesthetic exposure. The patient had pill rolling tremors and cog wheel rigidity at rest also .He was oriented to time, place and person. He was cooperative with history elicitation but showed mild signs of cognitive impairment. He was able perform his daily routine activities with assistance prior to the present illness.

On examination pallor was evident with mild dehydration. He was edentulous, with adequate mouth opening. Mallampatti grade III with decreased neck flexion and extension movements. His 
pulse rate was 136beats/min and it was regular with good volume. His blood pressure was 146/96 $\mathrm{mm} \mathrm{Hg}$ in supine posture left arm, patient`s respiratory rate was $36 \mathrm{cpm}$. Auscultation of his chest revealed bilateral crepitation.

His cardiovascular system examination was normal. His routine blood investigations, ECG and electrolytes were within normal limits. His chest X-ray showed prominent bronchovascular markings and COPD changes. Ultrasonography of his abdomen showed features of intestinal obstruction and grade II prostatic hypertrophy, with no organomegaly or ascites. The patient was accepted for anaesthesia with ASA grade III (E).

The patient was given his usual anti Parkinsonian medications via a nasogastric tube, one hour before the operation. Nebulization with salbutamol and ipratropium bromide was given pre operatively. Intra venous premedication was given with inj. glycopyrrolate $0.2 \mathrm{mg}$, inj. Ondansetron 4 mg, and inj. midazolam $1.5 \mathrm{mg}$. His monitoring included pulse oximetry, ECG, NIBP, ETCO2, temperature and urine output. We had difficulty in recording his basal vital parameters due to the tremors and rigidity.

A general anaesthesia with endotracheal intubation and controlled ventilation was planned. After preloading with $500 \mathrm{ml}$ of Ringer's lactate, pre oxygenation was done with $100 \%$ oxygen for 5 min. The patient was induced with inj. thiopentone $200 \mathrm{mg}$ iv and inj succinylcholine $75 \mathrm{mg}$ iv was given since patient's Mallampatti grading was III along with decreased neck flexion and extension anticipating difficult intubation, tracheal intubation was done with a $8.5 \mathrm{~mm}$ cuffed endotracheal tube.

The anaesthesia was maintained with $\mathrm{O}_{2}$ only + isoflurane $0.5 \%-1 \%+$ inj atracurium $25 \mathrm{mg}+5 \mathrm{mg}$. Fentanyl $50 \mathrm{mg}$ iv and inj paracetomol $750 \mathrm{mg}$ iv was used for the intra operative analgesia. The operative procedure, which lasted for three hours, consisted of the release of the adhesive bands in the ileal region. The intra operative urine output was $300 \mathrm{ml}$ and the blood loss was around $400 \mathrm{ml}$. Two liters of crystalloids were used intra operatively. The patient was aerodynamically stable throughout the procedure.

At the end of the surgery, patient was extubated, following a reversal with neostigmine and glycopyrrolate. His post-operative pain was managed with NSAIDs. His anti Parkinsonian medications were continued after surgery via a feeding tube for 2 days. He was then allowed to take them orally and he resumed his usual medication. His postoperative course was uneventful.

DISCUSSION: The degeneration of the dopaminergic neurons of the substantia nigra leads to PD. The autonomic dysfunction can manifest as orthostatic hypotension, leading to a sudden exaggerated response to the central neuraxial blockade. A delayed gastric emptying and thermoregulatory or genitourinary dysfunctions are common. The patients show altered responses to the vasopressors. 3,4,5

Upper airway dysfunction can cause retained secretions, atelectasis, aspiration, lower respiratory tract infections and post extubation laryngospasm. ${ }^{6}$

The Parkinsonism-Hyperpyrexia Syndrome (PHS) can occur in the perioperative period. It is characterized by high fever, extreme muscle rigidity, autonomic instability, altered consciousness, acute renal failure and a disseminated intravascular coagulation. PHS occurs in up to $4 \%$ of the PD patients; the mortality ranges from $4 \%$ for the treated to $20 \%$ for the untreated episodes. PHS can be prevented by an uninterrupted administration of medications. An early recognition and an aggressive treatment with fluid replacement, resuming of the dopaminergic therapy and supportive care, is necessary for a successful recovery. ${ }^{7}$ 
Dopa agonists can be given orally, intravenously or trans dermally. In the patients who are unable to take medications orally or through a gastric tube, parenterally administered anticholinergic drugs such as trihexyphenidyl, benztropine, or diphenhydramine can be given. Levodopa and carbidopa are absorbed in the small intestine. A duodenal feeding tube may be useful. ${ }^{3}$ Dopamine antagonists like droperidol, haloperidol, risperidone, metaclopramide, prochlorperazine, promethazine, phenothiazines, butyrophenones, etc should be avoided.

Regional anaesthesia has advantages over general anaesthesia as it avoids the effects of the general anaesthetics and the neuromuscular blocking drugs, which may mask the tremors. The high incidence of nausea and vomiting which is associated with general anesthesia prevents the effective administration of the oral medications and exacerbation can occur in the postoperative period. ${ }^{8}$

Thiopentone and propofol are relatively safe. Ketamine is contraindicated due to the exaggerated sympathetic responses which it produces. There are numerous reports of muscle rigidity following the use of fentanyl. ${ }^{9}$ Neuromuscular blocking agents can be used safely in patients with Parkinson's disease. Investigations which were done, have suggested that succinylcholine does not induce hyperkalaemia. ${ }^{10}$

Halothane, which sensitizes the heart to catecholamines, should be avoided in patients who are on Levodopa. Isoflurane is safe, as it does not sensitize the myocardium to catecholamines. Numerous case reports have described various approaches which were made to the perioperative management $t^{4,5}$, but there are no definitive treatment guidelines. The major goal of the management in the perioperative period is to continue the administration of the dopamine replacement therapy.

Our patient is also a case of diabetes mellitus. Diabetes mellitus can be of two type, type 1 and type 2 . Clinically type 2 diabetes represents $90-95 \%$ of all cases, leaving type 1 diabetes to account for the remaining $5-10 \% .{ }^{11}$ As compared to the non-diabetic population, diabetic patients have an increased rate of hospitalization frequency, longer hospital stays, and a greater number of ambulatory care visits. ${ }^{12}$

Hyperglycemia of diabetes is the consequence of relative or absolute deficiency of insulin and a relative or absolute excess of glucagon. In type 1 diabetes, there is an absolute deficiency in insulin production, and in type 2 diabetes is characterized by a relative deficiency in insulin, typically caused by insulin resistance.

\section{ANESTHETIC CONSIDERATION IN DIABETIC PATIENTS:}

- Diabetes affects oxygen transport by causing glucose to covalently bind to the hemoglobin molecule, decreasing oxygen saturation and red blood cell oxygen transport in diabetic patients. ${ }^{13}$

- One study ${ }^{14}$ demonstrated that diabetic patients with previously diagnosed autonomic dysfunction are at increased risk for intraoperative hypothermia. The pathogenesis may be related to inappropriate regulation of peripheral vasoconstriction to conserve body heat.

- Autonomic dysfunction also affects the body's ability to regulate blood pressure, leading to significant orthostatic hypotension. This underlying defect is caused by a lack of appropriate vasoconstriction. Denervation may also involve vagal control of the heart rate. The changes in heart rate seen with atropine and $\beta$-blockers are blunted in patients with significant autonomic dysfunction. ${ }^{15}$ 
- Men who suffer from diabetes have twice the age-adjusted risk for coronary artery disease. The risk for women is tripled, indicating that they may be even more sensitive to the cardiovascular effects of diabetes. ${ }^{16}$

- Diabetes affects the gastrointestinal tract in several ways. First, it damages the ganglion cells of the gastrointestinal tract, inhibiting motility, delaying gastric emptying and overall transit time through the gut. Theoretically, all diabetic patients have delayed gastric emptying and many practices treat these patients with the same considerations as patients with full stomachs. Thus, preoperative treatment with agents that inhibit acid secretion, neutralize stomach acid, and increase gastric emptying (e.g. famotidine, bicitra, and metoclopramide) is essential. Rapidsequence induction is commonly employed to try to minimize the risk of aspiration.

CONCLUSION: The patient of PD can be safely anesthetized, but a thorough perioperative assessment, minimizing the interruption of the drug therapy peri operatively and avoiding the known precipitating agents, are very important to reduce the postoperative mortality and morbidity.

\section{REFERENCES:}

1. DeLong MR, Juncos JL. Parkinson's disease and Other Movement Disorders. In: Kasper DL, Fauci AS, Longo DL, Braunwald E, Hauser SL, Jameson JL, editors. Harrison's Principles of Internal Medicine. 17th ed. New York: McGraw-Hill Medical Publishers; 2008. pp. 2406-17.

2. Rudra A, Rudra P, Chatterjee S, Das T, Ray M, Kumar P. Parkinson's Disease and Anaesthesia. Indian J Anaesth 2007; 51: 382.

3. Jankovic J, Complications and limitations of drug therapy for Parkinson's disease Neurology 2000; 55: S2-6.

4. Christopher B, Susan M, Calne RN, The management of medical and surgical problems in Parkinson's disease BCMJ 2001; 43 (4): 219-23.

5. Furuya R, Hirai A, Andoh T, Kudoh I, Okumura F, Successful perioperative management of a patient with Parkinson's disease by enteral levodopa administration under propofol anaesthesia Anaesthesiology 1998; 89: 261-63.

6. Easdown LJ, Tessler MJ, Minuk J, Upper airway involvement in Parkinson's disease resulting in postoperative respiratory failure Can J Anaesth 1995; 42: 344-47.

7. Nicholsan G, Pareira AC, Hall GM, Parkinsons disease and anesthesia Br J Anaesth 2002; 89: 904-16.

8. Shaikh SI, Verma H, Parkinson's disease and anaesthesia Indian J Anaesth 2011; 55: 228-34.

9. Comstock MK, Scamman FL, Carter JG, Moyers JR, Stevens WC, Rigidity and hypercarbia on fentanyl-oxygen induction Anaesthesiology 1979; 51: S28.

10. Muzzi DA, Black S, Cucchiara RF, The lack of effect of succinylcholine on serum potassium in patients with Parkinson's disease Anaesthesiology 1989; 71: 322.

11. CDC: National Diabetes Fact Sheet. United States; 2003.

12. US Preventive Services Task Force. Guidelines. In: Guide to Clinical Preventative Services. 2nd ed. St. Louis, MO; Williams and Wilkins; 1996, 193-208.

13. Madsen H, Ditzel J: Changes in red blood cell oxygen transport in diabetic pregnancy. Am J Obstet Gynecol; 1982, 143:421. 
14. Kitamura A, Hoshino T: Patients with diabetic neuropathy are at risk of a greater intraoperative reduction in core temperature. Anaesthesiology; 2000, 92: 1311.

15. T. sueda K, Huang KC, Dumond SW, et al: Cardiac sympathetic tone in anaesthetized diabetics. Can J Anaesth; 1991, 38: 20.

16. Kannel W, McGee D: Diabetes and cardiovascular disease: The Framingham Study. JAMA; 1979, 241: 2035.

\section{AUTHORS:}

1. Prajwal Patel H. S.

2. Shivaramu B. T.

3. Vidyadhar Metri

4. Amith S.

5. Kislaya Kumar

\section{PARTICULARS OF CONTRIBUTORS:}

1. Assistant Professor, Department of Anaesthesia, AIMS, Bellur.

2. Assistant Professor, Department of Anaesthesia, AIMS, Bellur.

3. Post Graduate, Department of Anaesthesia, AIMS, Bellur.

4. Post Graduate, Department of Anaesthesia, AIMS, Bellur.
5. Post Graduate, Department of Anaesthesia, AIMS, Bellur.

\section{NAME ADDRESS EMAIL ID OF THE CORRESPONDING AUTHOR:}

Dr. Vidyadhar Metri,

Final Year Anesthesia Post Graduate, Department of Anesthesia,

AIMS, B. G. Nagar,

Nagamangala Taluk -571448,

Mandya District, Karnataka.

Email: metrividyadhar@gmail.com

Date of Submission: 17/09/2014.

Date of Peer Review: 18/09/2014.

Date of Acceptance: 01/10/2014.

Date of Publishing: 09/10/2014. 